\title{
Diabetes mellitus among tuberculosis patients: a cross sectional study from Pakistan.
}

\author{
Zarfishan Tahir ${ }^{1,2}$, Mansur-ud-Din Ahmad², Abdul Majeed Akhtar ${ }^{3}$, Tahir Yaqub², \\ Muhammad Hassan Mushtaq ${ }^{2}$, Hasnain Javed ${ }^{1,4}$
}

1. Department of Bacteriology, Institute of Public Health, Lahore, Punjab, Pakistan.

2. University of Veterinary and Animal Sciences, Lahore, Punjab, Pakistan.

3. Provincial TB Control Program, Punjab, Pakistan.

4. Department of Microbiology and Molecular Genetics, University of the Punjab, Lahore, Pakistan.

\begin{abstract}
Background: The co-occurrence of diabetes mellitus (DM) and tuberculosis (TB) is largely associated with high frequency of morbidity.

Objective: To determine the prevalence of DM among TB patients and describe the socio-demographic and behavioral factors associated with TB-DM co-occurrence .

Methods: We enrolled 500 TB patients from September, 2014 to August 2015 at four major public sector hospitals of Lahore, Pakistan. A questionnaire was used to collect information regarding associated socio-demographic and behavioral factors of the patients. We monitored the fasting blood sugar of each patient by using a semi automated clinical chemistry analyzer followed by an HbA1c level check of all hyperglycemic patients.

Results: The prevalence of TB-DM co-occurrence was $14.8 \%$. The prevalence of TB-DM was higher $(62.2 \%)$ among males. The $>57$ year age group had the highest proportion of patients $(35.1 \%)$, with co-existent TB-DM. Most were illiterate $(73.0 \%)$ and unemployed (48\%). Moreover, among the 74 patients positive for TB-DM had a history of smoking. Age and education level were significantly associated with DM-TB while gender, occupation and smoking were not associated.

Conclusion: The study revealed a $14.8 \%$ prevalence of DM among TB patients. This was associated with several socio-demographic factors, including age, unemployment, literacy and polluted environment. Thus, poor and unhealthy lifestyles were the factors associated with DM among immunologically compromised individuals due to TB.
\end{abstract}

Keywords: Tuberculosis (TB), diabetes mellitus (DM), socio-demographic and behavioral factors.

DOI: http://dx.doi.org/10.4314/ahs.v16i3.5

Cite as: Tabir Z, Abmad M, Akbtar AM, Yaqub T, Mushtaq MH, Javed H. Diabetes mellitus among tuberculosis patients: a cross sectional study from Pakistan. Afri Health Sci 2016;16(3): 671-676. DOI: http://dx.doi.org/10.4314/abs.v16i3.5

\section{Introduction}

Diabetes mellitus (DM) is one of the most common diseases prevalent in low and middle income countries (LMICs) that are already facing problems due to various endemic infections like tuberculosis (TB) and malaria. The co-occurance of DM-TB is largely associated with higher frequency of morbidity, especially among LMICs that are facing epidemics of communicable and non-com-

\section{Correspondence author:}

Hasnain Javed,

Department of Microbiology and Molecular Genetics,

University of the Punjab, Lahore, Pakistan

Contact: +92-3334591434

Fax \# +92-4237500482

Email address: hasnain_javed@hotmail.com municable diseases ${ }^{1}$. According to the WHO, 371 million people are living with DM worldwide while $80 \%$ of them are living in LMICs. Moreover, these LMICs also bear $90 \%$ burden of worldwide TB patients. ${ }^{2}$ Several studies have reported that TB and DM are co-incident and may serve as pre-disposing risk factors for each other. In TB endemic areas, patients are particularly prone to DM when compared to healthy population ${ }^{3}$. Similarly, other studies have reported DM as a risk factor for TB. It is also reported that patients with TB-DM co-occurrence usually do not respond to anti-TB therapy thus resulting in higher mortality. ${ }^{4}$ Therefore, it is essential to study the prevalence of both diseases in order to understand the factors associated with their co-occurrance. ${ }^{5}$

Pakistan ranks fifth among 22 high burden TB countries and ranks fourth among 27 high burden drug resistant

African Health Sciences Vol 16 Issue 3, September 2016 
TB countries ${ }^{6}$. The World Health Organization (WHO) reported an estimated incindence of 275 cases per 100,000 persons and prevalence of 342 cases per 100,000 population ${ }^{7,8}$. In case of diabetes mellitus, the WHO has reported 4.3 million affected individuals in Pakistan and it is included among the top ten countries with highest DM incidence and it is expected that this figure will reach 14.5 million by the end of year 2025.' Therefore the Nation, having such a high burden of these two diseases, is at an increased risk of developing diabetes among TB infected patients. The exact mechanism of their inter-linkage is not clearly understood, but some studies suggested that reduced alveolar macrophage activity in TB patients is the main reason making them prone to $\mathrm{DM}^{10}$. On the other hand, diabetic patients have weak cellular immunity, kidney problems and alveolar angiopathy that ultimately make the conditions favorable for TB infection., ${ }^{2,4}$

Poverty and unhygienic life styles are the serious issues being faced by LMICs like Pakistan. These issues are making the situation more complicated and result in the co- occurrence of complex diseases like TB and DM. Therefore, there is an urgent need to develop some strategies regarding their control and prevention. Developing and effective strategy to address the issue needs sufficient information about the prevalence and spread of such diseases. The present study aimed to determine the frequency of DM among TB patients visiting four major hospitals of Lahore, Pakistan. It also determined the socio-demographic and behavioral factors associated with TB-DM co-occurrence.

\section{Materials and methods}

The present study was carried out at four major public sector hospitals of Lahore Metropolitan including Mayo hospital, Gulab Devi hospital, Services hospital and Infectious diseases hospital during a one year time period (September, 2014 to August, 2015). This cross sectional study received ethical approval from the board of ethics, Institute of Public Health, Lahore. A pre-tested comprehensive questionnaire covering different demographic and medical aspects of the present study was used to collect the information from the participants. Informed consent was taken from all individuals who participated in this study. All the filled questionnaires were analyzed for participants' socio-demographic, clinical and behavioral risk factors for DM and TB.

Almost $500 \mathrm{~TB}$ patients meeting the inclusion criteria were enrolled in this study. Patients with tuberculosis were characterized as those having positive results for TB disease manifestation by clinical, radiological, microbiological and molecular examinations ${ }^{11}$. Microbiological confirmation was done by Zeihl Neelsen staining meth$\mathrm{od}^{10}$ and culturing of Mycobacterium tuberculosis (MTB) was done on Lowen Stein Jensen (LJ) media by modified Petroff method ${ }^{12}$. Molecular confirmation was done using GeneXpert method. ${ }^{13}$ All microbiological and molecular assays were carried out at biosafety level 2 Provincial TB Reference Lab, Institute of Public Health, Lahore.

All study participants were further subjected to fasting blood sugar level by semi automated clinical chemistry analyzer Microlab 300 according to manufacturer's protocol. ${ }^{14}$ Patients having DM were categorized as those individuals who had fasting blood sugar level of higher than $126 \mathrm{mg} / \mathrm{dl}$ and HbA1C level $>6.8$ or if pre-diagnosed by physicians and fulfilling all criteria of American Diabetes Association to diagnose a patient with DM. ${ }^{15}$ Patients who had fasting blood sugar level more than $126 \mathrm{mg} / \mathrm{dl}$ were tested twice for two consective days for further confirmation. HbA1c level of all patients was also monitored according to guidelines suggested by American Diabetes Association (2015). Elevated HbA1c level (>6.5\%) is considered diagnostic marker for DM. HbA1c level was performed by Microlab-300 (ELITech Group) with randox reagent (Crumlin, County Antrim, United Kingdon) according to manufacturer's guidelines. ${ }^{16}$

Verification of the patient's particulars was done by comparing with the hospital data and the patients who had abnormal blood sugar level and elevated $\mathrm{HbA1C}$ level were further referred to the physicians for their treatment. Information about the identity of participating patients was not disclosed at any stage of the study thus all personal information of patients was kept confidential.

Statistical analysis, including, Chi square, odd ratios and $\mathrm{p}$ value were calculated using SPSS version17.0.

\section{Results}

During the one year study period, a total of 500 patients diagnosed with TB were recruited for the present study. Among them, 140 patients were screened from Infectious diseases hospital, 126 from Mayo hospital, 80 from Services hospital and 170 from Gulab Devi hospital were enrolled in this study. Out of the $500 \mathrm{~TB}$ patients, 74 $(14.8 \%)$ were found to be diabetic (Fig. 1). 


\section{Fig.1. Overall prevalence of diabetes among TB patients}

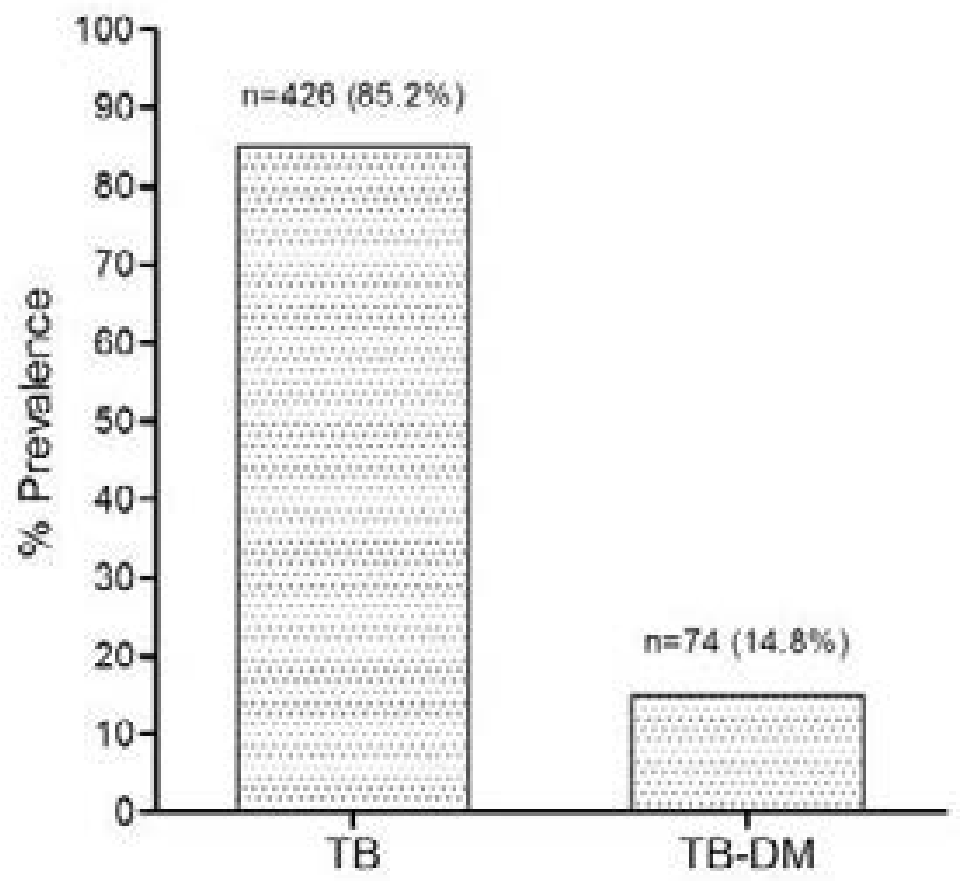

Among these 74 diabetic patients, 22 had been previously diagnosed with the disease, but their blood sugar level was $<126 \mathrm{mg} / \mathrm{dl}$ at the time of data collection. The remaining 52 patients were diagnosed during this study due to high blood sugar level $(>126 \mathrm{mg} / \mathrm{dl})$ and elevated HbA1c level $(>6.5 \%)$ and those were referred to the physician for further treatment.

Median age of the patients participating in the study was 38 years with the a range of $18-63$ years. While the patients having DM mostly belonged to the age group of $>57$ years $(n=26)$ only 4 patients were found in the $18-27$ years age group as shown in Table 1.

Among 500 patients that fulfilled the inclusion criteria, 340 or $68 \%$ were male. Similarly, among the 74 with TBDM co-occurrence, $62.16 \%$ (or 46) were male.

The statistical evaluation of socio-economic condition of the participants revealed that among the total participants, maximum number of patients $(n=220 ; 51.6 \%)$ were illiterate and a similar trend was observed in the case of known diabetic patients where $54(72.9 \%)$ individuals were illiterate (Table 1). Moreover, one of the socio-demographic factors studied was occupation of the participants that showed maximum co-incidence of TB-DM was spotted in house wives and unemployed individuals with the frequency of $32.43 \%$ each ( $n=24$ each). Broadly a significant difference between TB-DM and TB was observed in the category of age groups and education $(\mathrm{p} \leq 0.05)$ while no significant difference was observed in the category of gender, occupation and smoking ( $\mathrm{p} \geq 0.05$ )(Table 1).

Moreover, among diabetic patients, $27 \%(n=20)$ were known for their smoking history while the majority of these patients were non-smokers having frequency of $73 \%(n=54)$. Almost similar trend of smoking was observed in case of non-DM patients with TB. 
Table 1

\begin{tabular}{|c|c|c|c|c|c|c|}
\hline \multirow{2}{*}{ Variables } & \multirow{2}{*}{$\begin{array}{l}\text { TB-DM }(n=74) \\
\text { n (\%) }\end{array}$} & \multirow{2}{*}{$\begin{array}{l}\text { TB }(n=426) \\
n(\%)\end{array}$} & \multirow{2}{*}{ Odd ratio (OR) } & \multicolumn{2}{|c|}{ 95\% Confidence Interval } & \multirow{2}{*}{ *pvalue } \\
\hline & & & & Lower & Upper & \\
\hline Gender & & & - & & & 0.24 \\
\hline Male & $46(62.1)$ & $294(69)$ & 0.737 & .442 & 1.232 & 0.243 \\
\hline Female & $28(37.8)$ & $132(30.9)$ & - & - & & - \\
\hline Age groups (Yrs) & & & - & 40 & & 0.000 \\
\hline $18-27$ & $4(5.4)$ & $148(34.7)$ & 0.02 & 25.6 & & 0.000 \\
\hline $28-37$ & $6(8.1)$ & $72(16.9)$ & 0.08 & 3.7 & & 0.054 \\
\hline $38-47$ & $16(21.6)$ & $70(16.4)$ & 0.22 & 1.1 & & 0.270 \\
\hline $48-57$ & $22(29.7)$ & $62(14.5)$ & 0.35 & 10.3 & & 0.001 \\
\hline$>57$ & $26(35.1)$ & $74(17.3)$ & .35 & 12.4 & & 0.000 \\
\hline Education & & & - & 14.5 & & 0.005 \\
\hline Higher Secondary & $2(2.7)$ & $6(1.4)$ & 0.33 & 0.63 & & 0.400 \\
\hline Secondary & $2(2.7)$ & $46(107)$ & 0.04 & 4.75 & & 0.020 \\
\hline Illiterate & $54(72.9)$ & $220(51.6)$ & 0.24 & 11.5 & & 0.000 \\
\hline Middle & $0(0)$ & $6(1.4)$ & 0 & 1.05 & & 0.300 \\
\hline Primary & $16(21.6)$ & $148(34.7)$ & 0.10 & 4.9 & & 0.026 \\
\hline Occupation & & & - & 14.4 & & 0.070 \\
\hline Businessman & $10(13.5)$ & $58(13.6)$ & 0.17 & 0.000 & & 0.980 \\
\hline Butcher & $0(0)$ & $2(0.4)$ & 0 & 0.34 & & 0.550 \\
\hline Housewife & $24(32.4)$ & $98(23)$ & 0.20 & 3.03 & & 0.080 \\
\hline Labor & $8(10.8)$ & $60(14.1)$ & 0.13 & 0.57 & & 0.440 \\
\hline Milkman & $2(2.7)$ & $10(2.3)$ & 0.2 & 0.03 & & 0.850 \\
\hline None & $24(32.4)$ & $94(22)$ & 0.25 & 3.7 & & 0.052 \\
\hline Private Employee & $6(8.1)$ & $78(18.3)$ & 0.07 & 4.09 & & 0.030 \\
\hline Student & $0(0)$ & $20(4.6)$ & 0 & 3.6 & & 0.057 \\
\hline Veterinarian & $0(0)$ & $6(1.4)$ & 0 & 1.05 & & 0.30 \\
\hline Smoking & & & - & 0.43 & & 0.508 \\
\hline Smokers & $20(27)$ & $100(23)$ & 1.20 & .254 & 758 & 0.508 \\
\hline Non-smokers & $54(73)$ & $326(77)$ & - & - & & - \\
\hline
\end{tabular}

\section{Discussion}

LMICs are facing serious issues due to high burden of TB and emerging cases of multi-drug resistant tuberculosis (MDR TB). On the other hand DM has been declared as pandemic in the South Asian region. Furthermore the co-occurrence of these two diseases is creating further complication. This problem of DM-TB co-morbidity is mostly noticed in the LMICs because of endemic TB and DM caused by low socio-economic status and poor living $^{17 .}$ In this study we determined the prevalence of DM among TB patients of in Lahore, Pakistan. We also determined socio-demographic, clinical and other factors associated with the the co-occurrence of TB and DM. 
In the present study, out of 500 TB patients, 74 (14.8\%) were recorded as diabetic. Of these, 22 patients were already known DM and due to taking medicine they had fasting blood sugar level $<126 \mathrm{mg} / \mathrm{dl}$ at the time of data collection. The results of present study are similar to those of Amin et al. that reported co-occurrence of TB and DM in 14\% patients of Peshawar, Pakistan ${ }^{6}$. Similarly, another study of Pakistani population conducted by Jabbar et al. in 2006 showed co-occurrence of TB and DM among $11.9 \%$ study participants ${ }^{18}$.

Age of the patients participating in the present study ranged between 18-63 years while the median age was 38 years. However, most of the study participants who had co-occurrence of TB and DM belonged to the age group of $>57$ years with frequency of $35.13 \%$. Qayyum et al., reported that $77.7 \% \mathrm{DM}$ patients positive for $\mathrm{TB}$ tests were from age groups of $30-49$ years ${ }^{19}$. However, in the present study this pattern is quite varied and highest incidence has been observed among $>57$ years age group individuals that may be due to weak immune system along with multiple ailments. A study based on Ethiopian population conducted by Amare et al. displayed almost similar association of age and disease as encountered in this study ${ }^{20}$. Therefore, these results predict that the chances of TB-DM leading to co-morbidity increase significantly with age of the patient.

Higher incidence of DM in old age patients was also reported by the study of Baghaei et al. ${ }^{21}$. Analysis of the gender-based distribution revealed that male participants $(62.16 \%)$ were more affected by both of the diseases as compared to female $(37.83 \%)$. Similar results were reported by Patel et al. during his study conducted in Indian population that majority patients of TB-DM were male $(73.68 \%)$ while female were the minority $(26.32 \%) .{ }^{9}$ Moreover, similar results were obtained from another study conducted in Pakistan by Jabbar et al. revealed that $60.21 \%$ male patients out numbered the female participants $(39.78 \%)$ in terms of TB-DM co-incidence ${ }^{18}$. The perevelnce of higher ratio of TB-DM may be due to the case that male are exposed to the outer environment more as compared to female and encountered by multiple infections.

The socio-demographic factors being studied in this research included education and occupation. The results revealed that majority of the study subjects $(72.97 \%)$ were illiterate followed by those of primary class (21.62\%). While in terms of occupation, majority of the patients $(48 \%)$ were not doing any jobs and comprised of house wives and unemployed persons (24\% each). The results of present study are in agreement with those of Siddiqui et al. who described maximum prevalence of $\mathrm{TB}$ among illiterate and unemployed individuals with the frequencies of $29.60 \%$ and $20.4 \%$ respectively. ${ }^{22}$ Therefore, the study suggests that there is a strong correlation between disease manifestation and the status of employment along with literacy.

In the present study, we also studied the relation of smoking with TB-DM. We found the frequency of smoking was $27 \%$ and $23 \%$ in TB patients with and without DM respectively. Similar results were reported in Ethiopian population in the study conducted by Qayyum et al. where he found $28.57 \%$ frequency of smoking among DM-TB patients ${ }^{20}$. However, in the study of Siddiqui et al., $48.80 \%$ patients exhibited smoking that is a comparatively higher frequency of smoking observed than that in the present study. ${ }^{22}$

The present study indicated $14.8 \%$ prevalence of DM among TB patients that may serve as pre-disposing factor leading to the latent tubercle infection. Our study showed a significant relation between diabetes and different age groups, especially in old age group. Furthermore, the study revealed an augmenting impact of old age, sex, literacy and employment on the co-occurrence of TB-DM. Therefore, various socio-economic factors as well as behavioral aspects like smoking strongly influence such complicated diseases and there is need of awareness and education among common people to avoid such un healthy habits.

The study also revealed that $14.8 \%$ DM-TB prevalence in patients which confirms the fact that people with TB, must be appropriatly screened for DM.

\section{Conflict of interest}

The authors declare no conflict of interest.

\section{References}

1. Kibirige D, Ssekitoleko R, Mutebi E, Worodria W. Overt diabetes mellitus among newly diagnosed Ugandan tuberculosis patients: a cross sectional study. $B M C$ Infectious Diseases. 2013;13(1):122.

2. Harries A, Satyanarayana S, Kumar A, Nagaraja S, Isaa- 
kidis P, Malhotra S, et al. Epidemiology and interaction of diabetes mellitus and tuberculosis and challenges for care: a review. Public Health Action. 2013;3(Suppl 1):S3

3. Ottmani S, Murray M, Jeon C, Baker M, Kapur A, Lönnroth $\mathrm{K}$, et al. Consultation meeting on tuberculosis and diabetes mellitus: meeting summary and recommendations [Meeting report]. The International Journal of Tuberculosis and Lung Disease. 2010;14(12):1513-7.

4. Dooley KE, Chaisson RE. Tuberculosis and diabetes mellitus: convergence of two epidemics. The Lancet Infectious Diseases. 2009;9(12):737-46.

5. Kermansaravi F, Metanat M, Sharifi-Mood B. Evaluation of active pulmonary tuberculosis among patients with diabetes. Int J Infect. 2014;1(1):e19632.

6. Mustafa S, Javed H, Hashmi J, Jamil N, Tahir Z, Akhtar AM. Emergence of mixed infection of Beijing/Non-Beijing strains among multi-drug resistant Mycobacterium tuberculosis in Pakistan. 3Biotech. 2016; 6(1), 1-9.

7. Javed H, Tahir Z, Hashmi HJ, Jamil N. A cross-sectional study about knowledge and attitudes toward multidrugresistant and extensively drug-resistant tuberculosis in a high-burden drug-resistant country. International Journal of Mycobacteriology. 2016; 5(2), 128-134

8. Javed H, Jamil N. Genotypes and drug resistance patterns of multi-drug-resistant Mycobacterium tuberculosis strains in Pakistan. European Respiratory Journal. 2015; 46(suppl 59), p.PA2687.

9. Fatima R, Qadeer E, Hinderaker S, Yaqoob A, Enarson $\mathrm{D}$, Bassili A, et al. Can the number of patients with presumptive tuberculosis lost in the general health services in Pakistan be reduced? The International Journal of Tuberculosis and Lung Disease. 2015;19(6):654-6.

10. Amin S, Khattak MI, Shabbier G, Wazir MN. Frequency of pulmonary tuberculosis in patients with diabetes mellitus. Gomal Journal of Medical Sciences. 2012;9(2). 11. Patel AK, Rami KC, Ghanchi F. Clinical Profile of Sputum Positive Pulmonary Tuberculosis Patients with Diabetes Mellitus in a Teaching Hospital at Jamnagar, Gujarat. National Journal of Medical Research. 2012;2(3):30912.

12. Abou-Seif MA, Youssef A-A. Evaluation of some biochemical changes in diabetic patients. Clinica Chimica Acta. 2004;346(2):161-70.
13. Myneedu VP, Singhal R, Khayyam KU, Sharma PP, Bhalla M, Behera D, et al. First and second line drug resistance among treatment naïve pulmonary tuberculosis patients in a district under Revised National Tuberculosis Control Programme (RNTCP) in New Delhi. Journal of epidemiology and global health. 2015; 5(4),365-373.

14. Boehme CC, Nicol MP, Nabeta P, Michael JS, Gotuzzo E, Tahirli R, et al. Feasibility, diagnostic accuracy, and effectiveness of decentralised use of the Xpert MTB/ RIF test for diagnosis of tuberculosis and multidrug resistance: a multicentre implementation study. The Lancet. 2011;377(9776):1495-505.

15. Zain M, Awan FR, Amir S, Baig SM. A case control association study of COMT gene polymorphism (I/D) with type 2 diabetes and its related factors in Pakistani Punjabi population. Journal of Diabetes \& Metabolic Disorders. 2015;14(1):40.

16. Association AD. 2. Classification and diagnosis of diabetes. Diabetes Care. 2015;38(Supplement 1):S8-S16.

17. Berwick M. Prediabetes: The Variation between HbA1c and Fasting Plasma Glucose. Int J Diabetol Vasc Dis Res S. 2015;2:1-7.

18. Alladin B, Mack S, Singh A, Singh C, Smith B, Cummings E, et al. Tuberculosis and diabetes in Guyana. International Journal of Infectious Diseases. 2011;15(12):e818-e21. 19. Jabbar A, Hussain S, Khan A. Clinical characteristics of pulmonary tuberculosis in adult Pakistani patients with co-existing diabetes mellitus. Eastern Mediterranean Health Journal. 2006; 12(5), 523.

20. Qayyum M, Shafiq M, Farogh A. Prevalence of pulmonary tuberculosis among diabetics. Biomedica. 2004;20(1):74-8.

21. Amare H, Gelaw A, Anagaw B, Gelaw B. Smear positive pulmonary tuberculosis among diabetic patients at the Dessie referral hospital, Northeast Ethiopia. Infect Dis Poverty. 2013;2(6).

22. Baghaei P, Marjani M, Javanmard P, Tabarsi P, Masjedi MR. Diabetes mellitus and tuberculosis facts and controversies. J Diabetes Metab Disord. 2013;12(1):58.

23. Siddiqui MS, Fakih HAM, Burney WA, Iftikhar R, Khan N. Environmental and Host-related Factors Predisposing to Tuberculosis in Karachi: A Cross-sectional Study. Journal of Pioneering Medical Sciences. 2011;1(1):13. 